\title{
Composición corporal, somatotipo y condición física en mujeres deportistas colombianas
}

\section{Body composition, somatotype and physical condition in colombian women athletes}

\author{
Carlos Alberto Ramos-Parrací ${ }^{1}$ (D); Felipe Augusto Reyes-Oyola ${ }^{1}$ (D); Constanza Palomino-Devia ${ }^{1}$ (D)
}

1Universidad del Tolima, Ibagué, Colombia.caramosp@ut.edu.co; fareyeso@ut.edu.co; cpalominod@ut.edu.co

Cómo citar: Ramos-Parrací, C.A.; Reyes-Oloya, F.A.; Palomino-Devia, C. 2021. Composición corporal, somatotipo y condición física en muje-res deportistas colombianas. Rev. Digit. Act. Fis. Deport. 7(2):e1852. http:// doi.org/10.31910/rdafd.v7.n2.2021.1852

Artículo de acceso abierto publicado por Revista Digital: Actividad Física y Deporte, bajo una licencia Creative Commons CC BY-NC 4.0

Publicación oficial de la Universidad de Ciencias Aplicadas y Ambientales U.D.C.A, Institución de Educación Superior Acreditada de Alta Cali-dad por el Ministerio de Educación Nacional.

Recibido: marzo 2 de 2021 Aceptado: marzo 23 de 2021 Editado por: Álvaro José Gracia Díaz

\section{RESUMEN}

Introducción: El propósito del estudio fue determinar la composición corporal, somatotipo y condición física en deportistas colombianas. Objetivo: Comparar estos componentes en función de la edad y asociar las variables estudiadas. Metodología: Participaron 160 mujeres de 10 modalidades deportivas, con rango de 14 a 22 años $(18,01 \pm 2,65)$. Se les realizó prueba de laboratorio indirecta en tapiz rodante de resistencia cardiovascular, Test de Squat Jump (SJ) y Salto contramovimiento (CMJ), Test de Wells (Sit and Reach), Test de resistencia de musculatura abdominal en 1 minuto. Para la toma de datos cinentropométricos, se utilizaron las técnicas de medición, recomendadas por la Sociedad Internacional para el Avance de la Cineantropometría, registrándose un total de 20 medidas. Resultados: El análisis cuantitativo permitió establecer que en las deportistas prevaleció la masa muscular y el somatotipo mesomórfico. Las comparaciones por grupos de edad evidenciaron que el IMC, la flexibilidad y la fuerza de resistencia abdominal fue mayor en el grupo de menor edad, mientras que el porcentaje de grasa, el SJ, el CMJ y la capacidad aeróbica fue mejor en el grupo de mayor edad; hubo correlaciones negativas entre del IMC con la capacidad aeróbica y los saltos y el ectomorfismo con la flexibilidad $(p<0,05)$. Conclusiones: De acuerdo con los resultados del estudio es fundamental conocer, objetivamente, el rendimiento físico de las deportistas y su composición corporal, para marcar los objetivos de la planificación del entrenamiento y programas de nutrición.

Palabras clave: Mujer; Composición corporal; Deportista; Antropometría.

\section{ABSTRACT}

Introduction: The purpose of the study was to determine the body composition, somatotype and physical condition in Colombian athletes. Objective: To compare these components based on age and associate the variables studied. Methodology: 160 women from 10 sports modalities participated, with a range of 14 to 22 years $(18.01 \pm 2.65)$. Indirect laboratory tests were performed on a treadmill for cardiovascular resistance, Squat Jump Test (SJ) and Countermovement Jump (CMJ), Wells Test (Sit and Reach), Abdominal Muscle Endurance Test in 1 minute. For kinenthropometric data collection, the measurement techniques recommended by 
the International Society for the Advancement of Kineanthropometry were used, recording a total of 20 measurements. Results: The quantitative analysis made it possible to establish that the muscle mass and the mesomorphic somatotype prevailed in the athletes. Comparisons by age group showed that BMI, flexibility and abdominal resistance strength were higher in the younger group, while the percentage of fat, SJ, CMJ and aerobic capacity were better in the group of Older; there were negative correlations between BMI with aerobic capacity and jumping, and ectomorphism with flexibility $(p<0.05)$. Conclusions: According to the results of the study, it is essential to objectively know the physical performance of the athletes and their body composition in order to mark the objectives of the planning of training and nutrition programs.

Keywords: Woman; Body composition; Athlete; Anthropometry.

\section{INTRODUCCIÓN}

Al hablar de condición física, se determina que, por medio de pruebas de laboratorio y de campo, se puede controlar los progresos y la prescripción del rendimiento, como lo menciona Sánchez \& Salas (2009). Teniendo en cuenta que el rendimiento en los deportistas es una combinación de diversos factores es muy posible que el factor más importante, a la hora de terminar el potencial de un deportista, sea la dotación genética. Otro aspecto que se debe mencionar, debido a que tiene un efecto sobre el rendimiento es la idoneidad y la cantidad de entrenamiento, previo a las competiciones.

De esta forma, resulta importante obtener información objetiva sobre el rendimiento físico de los deportistas con el fin de poder marcar objetivos de formación, programas de intervención a corto y largo plazo o simplemente información que sirve de motivación para entrenar en mejores condiciones según Irigoyen \& Arcos Larumbe (2013). Esta información, como lo menciona Irigoyen \& Arcos Larumbe (2013), se puede conseguir mediante el uso de pruebas, que evalúan la capacidad de rendimiento físico y los test de campo, que permiten su implementación a un gran número de deportistas de forma simultánea y rápida, debido a que son, generalmente, más baratos y fáciles de administrar, que las pruebas de laboratorio.
No se debe olvidar que las capacidades físicas son propias de cada individuo, presentan una influencia genética y se desarrollan mediante el ejercicio. Moro et al. (2016), las denominan como cualidades físicas y las definen como los factores que determinan la condición física del sujeto, que lo orientan hacia la realización de una determinada actividad y posibilitan el desarrollo de su potencial físico, mediante su entrenamiento.

A nivel deportivo, García et al. (2014) mencionan que la cineantropometría permite evaluar la composición corporal, morfológica, estado nutricional y proporciones del deportista y con esta información, orientar los parámetros hacia un máximo rendimiento, en su disciplina deportiva; también permite comprobar en los deportistas la simetría de su desarrollo corporal y detectar, a tiempo, posibles desviaciones en el aparato locomotor.

De otra parte, se debe mencionar que el control y la prevención de la desnutrición es una tarea inconclusa en Colombia, como en otros países; las enfermedades que se generan con esta situación son una de las principales causas de muerte, a nivel global. Los cambios en los estilos de alimentación, los patrones de trabajo y la falta de actividad física están causando sobrepeso, obesidad, diabetes, hipertensión y enfermedades cardiovasculares, en los países más pobres. En Colombia, como en otras naciones, se han utilizado criterios antropométricos, como el peso, la talla y el índice de masa corporal, para definir la obesidad, la desnutrición o trastornos alimentarios, referenciado en Nutrition and Health Transition in the Developing World: The Time to Act (2002).

Para Martínez et al. (2005), los cambios morfológicos de niños, jóvenes y adultos están sujetos a cambios constantes, durante el transcurso de la vida, debido a que en los estilos de vida pueden conducir a la presencia de factores de riesgo en enfermedades crónicas, asociados con un mayor grado de riesgo de eventos adversos a la salud y una mayor mortalidad.

Sedebe recordar, comolo menciona García etal.(2014), que el grupo español de cineantropometría GREC y la ISAK, elaboraron los estándares internacionales de comparación antropométrica, para medir los pliegues, los perímetros y los diámetros y, de esta forma, establecer el somatotipo del deportista, desde las etapas de formación hasta el máximo rendimiento 


\section{MATERIALES Y MÉTODOS}

deportivo, comparando, de esta manera, al deportista consigo mismo y con los deportistas de mayor éxito, en su deporte.

Por lo expuesto, el objetivo central de este estudio fue determinar la composición corporal, somatotipo y condición física en deportistas colombianas, así como comparar estos componentes, en función de la edad y buscar asociación entre las variables estudiadas.
Enfoque, tipo de estudio y participantes. La presente investigación, se realizó bajo un enfoque cuantitativo, de corte transversal y tipo de estudio descriptivo y correlacional. Participaron, voluntariamente, un total de 160 deportistas femeninas, pertenecientes a 10 modalidades deportivas, de diferentes ligas, del Departamento del Huila (Colombia) (Tabla 1), con edades entre los 14 a 22 años $(18,01 \pm 2,65)$.

Tabla 1. Distribución de las participantes de acuerdo a la modalidad deportiva.

\begin{tabular}{|c|c|c|}
\hline \multirow{2}{*}{ Grupo } & Deporte & $\begin{array}{c}\text { Deportistas } \mathbf{n} \\
(\mathbf{\%})\end{array}$ \\
\hline \multirow{2}{*}{ Deportes de combate } & Lucha & $3(1,9)$ \\
\hline \multirow{2}{*}{ Deportes con pelotas } & Taekwondo & $2(1,3)$ \\
\cline { 2 - 3 } & Tenis de campo & $3(1,9)$ \\
\cline { 2 - 3 } Deportes de tiempo y marca & Baloncesto & $14(8,8)$ \\
\cline { 2 - 3 } & $\begin{array}{c}\text { Levantamiento de } \\
\text { pesas }\end{array}$ & $11(6,9)$ \\
\cline { 2 - 3 } & Natación & $12(7,5)$ \\
\cline { 2 - 3 } & Natación sub- acuática & $54(33,8)$ \\
\cline { 2 - 3 } & Patinaje & $12(7,5)$ \\
\hline Deportes de arte y precisión & Canotaje & $22(13,8)$ \\
\hline Total & Gimnasia & $160(100)$ \\
\hline
\end{tabular}

\section{Instrumentos y procedimiento.}

Medidas antropométricas y sociodemográficas. La edad, el sexo y las medidas antropométricas fueron consignadas en una hoja de registro diseñada para tal fin, siguiendo los protocolos establecidos por la ISAK (2001). Se tomó el peso (Báscula SECA 700), la talla (Estadiómetro portátil WCS - Modelo WOOD Brasil), los pliegues cutáneos de tríceps, subescapular, suprailíaco, abdominal, muslo anterior, medial pierna (Calibrador CESCORF - Modelo Científico - Brasil); los diámetros óseos biacromial, bicrestal, húmero, muñeca, fémur (Calibrador para diámetros pequeños - Modelo FAGA S.R.L-Brasil) y perímetros musculares de brazo relajado, brazo contraído, antebrazo, muñeca, cintura, cadera, muslo superior, pantorrilla (Cinta Antropométrica - WISO modelo R88 - Brasil).

Composición corporal y somatotipo. Se obtuvo el índice de masa corporal (IMC), mediante la ecuación masa $(\mathrm{kg}) /$ talla $\left(\mathrm{m}^{2}\right)$, detallada en Ramos \& Gómez (2018). Luego, se calculó el porcentaje de grasa, el porcentaje de masa muscular, el porcentaje de masa residual y el porcentaje de masa ósea; también se determinó el somatotipo de cada deportista (Ramos \& Gómez, 2018; Sterkowicz et al. 2019).

Condición física. Para la resistencia cardiovascular, el evaluado corrió en la Banda Rodante hasta alcanzar su frecuencia cardiaca máxima teórica, hallada mediante la fórmula de Tanaka et al. (2001), para deportistas: FC máx = 205-(0,6 x Edad). La prueba, se realizó mediante el protocolo establecido por Ramos \& Gómez (2018).

En cuanto a la resistencia de la musculatura abdominal, se utilizó el Test de Abdominales en 1 min, relacionado en Silva et al. (2006). Respecto a la flexibilidad, se halló mediante la prueba de Sit And Reach (Wells \& Dillon, 1952).

Los test de SJ y CMJ, se ejecutaron en la plataforma de contacto, marca AxonJump " $\mathrm{T}$ "- Software Axon Bioingeniería Deportiva 4.02. Ambos saltos siguieron el protocolo establecido en Balsalobre et al. (2012).

Análisis de datos. Los análisis descriptivos e inferenciales fueron realizados en el programa 
estadístico IBM SPSS versión 25. La estadística descriptiva incluyó el informe de la media y la desviación estándar de las diferentes variables. Por su parte, en la estadística inferencial, se efectúo la prueba de análisis de varianza ANOVA de un factor, con una significación estadística de $p<0,05$; las comparaciones a posteriori, se obtuvieron con la prueba de Tukey. Para determinar las relaciones entre las variables, se empleó el coeficiente de correlación de Pearson (r), con un nivel de significancia de $p<0,05$.

Consideraciones éticas. Los test fueron realizados previo consentimiento informado de las participantes. El estudio, se rigió bajo la Resolución 008430 de
1993, emitida por el Ministerio de Salud de Colombia (Ministerio de Salud, 1993) y las normas deontológicas reconocidas por la declaración de Helsinki (WMA, 2017).

\section{RESULTADOS Y DISCUSIÓN}

La tabla 2 muestra la media y la desviación estándar de las variables básicas, de composición corporal, de somatotipo y de condición física, de la muestra de deportistas colombianas examinadas. En las participantes, se evidencia una prevalencia del porcentaje de masa muscular y, de esta manera, en el somatotipo predomina el componente mesomórfico.

Tabla 2. Variables básicas, composición corporal, somatotipo y condición física de las deportistas colombianas.

\begin{tabular}{|c|c|c|}
\hline \multicolumn{2}{|c|}{ Variables } & $\mathrm{X} \pm \mathrm{DE}^{\beta}$ \\
\hline \multirow{4}{*}{ Variables básicas } & Edad (años) & $18,01 \pm 2,65$ \\
\hline & Estatura $(\mathrm{cm})$ & $1,60 \pm 0,73$ \\
\hline & Peso (kg) & $56,17 \pm 9,38$ \\
\hline & $\operatorname{IMC}\left(\mathrm{kg} / \mathrm{m}^{2}\right)^{\infty}$ & $21,80 \pm 2,74$ \\
\hline \multirow{4}{*}{ Composición corporal } & Grasa (\%) & $12,95 \pm 3,14$ \\
\hline & Músculo (\%) & $48,09 \pm 32,91$ \\
\hline & Residual (\%) & $21,01 \pm 0,68$ \\
\hline & Óseo (\%) & $21,27 \pm 14,61$ \\
\hline \multirow{3}{*}{ Somatotipo } & Endomorfía & $3,04 \pm 1,0$ \\
\hline & Mesomorfía & $4,42 \pm 1,37$ \\
\hline & Ectomorfía & $3,14 \pm 5,75$ \\
\hline \multirow{5}{*}{ Condición física } & $\mathrm{VO}_{2 \max }(\mathrm{ml} . \mathrm{kg} \cdot \min )^{\pi}$ & $35,49 \pm 10,98$ \\
\hline & $\mathrm{SJ}(\mathrm{cm})^{\neq}$ & $28,17 \pm 8,32$ \\
\hline & $\mathrm{CMJ}(\mathrm{cm})^{\mu}$ & $32,16 \pm 8,46$ \\
\hline & Flexibilidad (cm) & $34,57 \pm 10,31$ \\
\hline & FRA (repeticiones) & $65,93 \pm 24,11$ \\
\hline
\end{tabular}

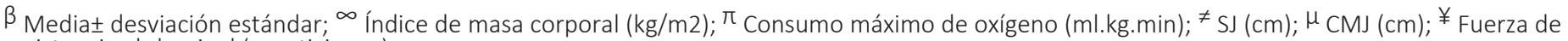
resistencia abdominal (repeticiones).

Respecto a los resultados de la variable talla $(1,70 \mathrm{~m}$ vs $1,60 \mathrm{~m})$, peso $(62,93 \mathrm{~kg}$ vs $56,17 \mathrm{~kg})$ y grasa $(27,08 \%$ vs $12,95 \%)$, respectivamente, se encontraron datos más altos en el estudio realizado por Gutiérrez et al. (2020), que se realizó con 6 mujeres, con una media de edad $(18,66 \pm 2,58$ años). Las deportistas, se encontraban concentradas en el centro de entrenamiento olímpico (CEO), ubicado en Curuma, en la región de Valparaíso en Chile; el estudio, se realizó previo al clasificatorio Panamericano Lima 2019. Así como en el estudio de Castiblanco et al. (2020), se evidenciaron datos más altos en la variable peso $(58,53 \mathrm{~kg}$ y $62,1 \mathrm{~kg})$ y en el porcentaje de grasa, en estudios, como el de Castiblanco et al. (2020) y Rivera et al. (2017), con valores de $19,01 \%$ y $24,34 \%$, respectivamente.
De la misma manera, se encontraron los resultados similares con relación a las mismas variables $(1,65 \mathrm{~m}$, $74,9 \mathrm{~kg}$ y $29,3 \%)$ y $(1,65 \mathrm{~m}, 61,51 \mathrm{~kg}$ y $24,51 \%)$, respectivamente, estudio efectuado por González et al. (2011), tomando como muestra 11 bailadoras de flamenco de la provincia de Cádiz, que habían participado en representaciones artísticas de carácter regional y local y González et al. (2015), donde la población de estudio estuvo constituida por 17 mujeres, entre 16 y 37 años, jugadoras del equipo semiprofesional femenino de Torrelodones C.F., perteneciente a la Segunda División Femenina de España. 
Del mismo modo, al comparar lo encontrado con una investigación realizada con 190 jugadoras de fútbol, la talla obtuvo resultados similares $(1,61 \mathrm{~m})$ (Sedano et al. 2009). También, en una muestra de 53 jóvenes universitarios colombianos, que hacían parte de una modalidad deportiva de conjunto (fútbol, fútbol sala o baloncesto), que representan cada una de las universidades en Manizales, se encontraban entre los 20 y 23 años; asimismo, la mayoría de los evaluados, se hallan en los primeros tres semestres de pregrado $(1,62 \mathrm{~m})$, descritos por Castiblanco et al. (2020); pero en el caso del peso y el porcentaje de grasa, las deportistas españolas obtuvieron datos más altos $(59,54 \mathrm{~kg}$ vs $56,17 \mathrm{~kg})$ y $(25,61 \%$ vs $12,95 \%)$, respectivamente, mencionados por Sedano et al. (2009), cuya muestra era de 190 jugadoras de fútbol, pertenecientes a equipos inscritos en Primera División Regional Femenina de Castilla y León $(n=90)$ y en Primera División Nacional Femenina $(n=100)$.

Por el contrario, una muestra de 19 jugadoras de fútbol soccer, pertenecientes al equipo representativo de una Universidad del Norte de México, en la temporada competitiva, obtuvieron datos más bajos en la talla y en el peso $(1,59 \mathrm{~m}$ y $55,6 \mathrm{~kg})$, aclarando que la edad promedio fue de 20,6 y la muestra fue más pequeña. Las jugadoras fueron clasificadas por su posición en el campo, en tres categorías: defensas, medias y delanteras (Caballero et al. 2019).

Respecto al IMC, estudios como el de Rivera et al. (2017), donde participaron en la investigación 53 estudiantes universitarios de primer año, con un promedio de 19,25 años de edad, 30 eran de la carrera de Pedagogía en Educación Física y 23 de Nutrición y Dietética, de la Universidad de La Frontera, en el 2014 y el de García et al. (2020), con una muestra de cadetes valorados en el Centro de Investigaciones de la Cultura física (CICFI), en la Escuela Militar de Cadetes "General José María Córdova” (Bogotá, Colombia), que fueron valorados por el servicio de nutrición deportiva y el laboratorio cardiopulmonar de la institución, cuyos resultados presentaron datos más altos, con relación al estudio de las deportistas colombianas $(24,38 \mathrm{~kg} /$ $m^{2}$ y $\left(27,4 \mathrm{~kg} / \mathrm{m}^{2}\right.$, respectivamente).

Caso contrario, en los estudios de Castiblanco et al. (2020) y González et al. (2015), cuya muestra era de 124 mujeres, pertenecientes a 28 deportes del Centro de Alto Rendimiento de Santiago (CAR), donde sus resultados fueron similares en la misma variable $\left(21,94 \mathrm{~kg} / \mathrm{m}^{2}-22,81 \mathrm{~kg} / \mathrm{m}^{2}-22,9 \mathrm{~kg} / \mathrm{m}^{2}\right)$; lo mismo se encontró los estudios de Palomino et al. (2016) $\left(20,4 \mathrm{~kg} / \mathrm{m}^{2}\right) \quad$ (aclarando que eran jóvenes escolares, con una muestra de 614 mujeres) y la investigación de Palomino et al. (2018) $\left(20,54 \mathrm{~kg} / \mathrm{m}^{2}\right)$, cuya muestra fue mayor, con 652 mujeres.

En cuanto al porcentaje muscular, los datos encontradosfueron más bajos en estudios, como el de Gutiérrez et al. (2020), Sedano et al. (2009), Castiblanco et al. (2020) y González et al. (2015), en comparación con las deportistas colombianas (48,09\% vs $45,68 \%, 38,79 \% \quad 44,48 \%$ y $43,64 \%)$, citados por Gutiérrez et al. (2020). Asimismo, el porcentaje residual (9,92\%) y el porcentaje óseo (11,23\% y 14,68\%), nombrados por Gutiérrez et al. (2020) y Sedano et al. (2009).

Respecto al somatotipo, en el estudio de Gutiérrez et al. (2020), se encontraron los datos más bajos, en el componente de endomorfia. El mismo estudio, también tuvo resultados más bajos en mesomorfia y ectomorfia, así como se evidenciaron en los resultados de los estudios de Sedano et al. (2009) y Caballero et al. (2019). Por el contrario, los resultados de la endomorfia fueron más altos, en las mismas investigaciones.

Frente al estudio realizado por González et al. (2011), ejecutaron el test del Escalón de McArdle, que ofrece una estimación del $\mathrm{VO}_{2}$ máx $(36,99 \mathrm{ml} / \mathrm{kg} / \mathrm{min})$; de la misma manera, el estudio realizado por Rivera et al. (2017), con estudiantes universitarias $(34,67 \mathrm{ml} /$ $\mathrm{kg} / \mathrm{min}-36,99 \mathrm{ml} / \mathrm{kg} / \mathrm{min})$, se proporcionaron datos similares, con relación al presente estudio.

Con respecto a los test SJ y CMJ, se evidenciaron datos más bajos en las bailadoras españolas de flamenco SJ $(22,53 \mathrm{~cm}$ vs $28,17 \mathrm{~cm})$ y CMJ $(25,11 \mathrm{~cm}$ vs $32,16 \mathrm{~cm})$, encontrados por González et al. (2011). Los resultados muestran datos muy similares en las variables relacionadas con la composición corporal y el VO2máx y datos más altos en los componentes del somatotipo, en las deportistas colombianas.

\section{CONCLUSIONES}

Las comparaciones de las variables en las deportistas, de acuerdo con el grupo de edad, se presentan en la tabla 3. Respecto a la composición corporal, el grupo de menor edad mostró mayores cifras en el IMC y el porcentaje de masa residual, el grupo de 17 a 19 años 
obtuvo valores más altos, en porcentaje de grasa y en porcentaje óseo, mientras que el grupo de mayor edad, tuvo mejor porcentaje de masa muscular; en dichas variables, las diferencias no fueron significativas $(p>0,05)$. En cuanto al somatotipo, el endomorfismo, mesomorfismo y ectomorfismo fue mayor en el grupo de 17 a 19 años, sin diferencias estadísticamente significativas $(p>0,05)$.

Tabla 3. Comparaciones de las variables de estudio en las deportistas diferenciadas por grupos de edad.

\begin{tabular}{|c|c|c|c|c|}
\hline \multirow[t]{2}{*}{ Variables } & $\begin{array}{c}14-16 \text { años } \\
(n=52)\end{array}$ & $\begin{array}{c}17-19 \text { años } \\
(n=51)\end{array}$ & $20-22$ años $(n=57)$ & \multirow[t]{2}{*}{$p^{\S}$} \\
\hline & $X \pm D^{\beta}$ & $X \pm D E^{\beta}$ & $X \pm D E^{\beta}$ & \\
\hline $\operatorname{IMC}\left(\mathrm{kg} / \mathrm{m}^{2}\right)^{\infty}$ & $22,13 \pm 2,95$ & $21,80 \pm 2,41$ & $21,50 \pm 2,83$ & 0,491 \\
\hline Grasa (\%): & $12,74 \pm 3,40$ & $13,58 \pm 2,83$ & $12,57 \pm 3,12$ & 0,214 \\
\hline Músculo (\%): & $45,09 \pm 6,41$ & $45,65 \pm 3,33$ & $53,02 \pm 54,67$ & 0,372 \\
\hline Residual (\%): & $21,07 \pm 0,75$ & $20,90 \pm 0,00$ & $21,05 \pm 0,89$ & 0,366 \\
\hline Óseo (\%): & $20,29 \pm 2,20$ & $22,94 \pm 25,74$ & $20,66 \pm 2,51$ & 0,610 \\
\hline Endomorfía: & $2,99 \pm 1,09$ & $3,19 \pm 0,94$ & $2,94 \pm 0,96$ & 0,402 \\
\hline Mesomorfía: & $4,26 \pm 1,32$ & $4,57 \pm 1,44$ & $4,44 \pm 1,36$ & 0,522 \\
\hline Ectomorfía: & $2,58 \pm 1,33$ & $4,41 \pm 9,94$ & $2,50 \pm 1,38$ & 0,158 \\
\hline$V O_{\text {máx }}(\mathrm{ml} . \mathrm{kg} . \min )^{\pi}$ & $32,63 \pm 11,33^{a}$ & $35,71 \pm 10,55$ & $37,89 \pm 10,61^{\mathrm{a}}$ & 0,042 \\
\hline SJ $(\mathrm{cm})^{\neq}$ & $27,68 \pm 6,63$ & $26,97 \pm 6,27$ & $29,68 \pm 10,85$ & 0,211 \\
\hline $\mathrm{CMJ}(\mathrm{cm})^{\mu}$ & $31,66 \pm 6,79$ & $31,07 \pm 6,43$ & $33,60 \pm 11,0$ & 0,265 \\
\hline Flexibilidad $(\mathrm{cm})$ : & $36,65 \pm 9,72$ & $32,44 \pm 11,74$ & $34,58 \pm 9,19$ & 0,117 \\
\hline FRA $\left(\right.$ rep) ${ }^{*}$ & $69,88 \pm 26,81$ & $61,49 \pm 23,34$ & $66,31 \pm 21,83$ & 0,209 \\
\hline
\end{tabular}

$\beta$ Media \pm desviación estándar; ${ }^{\infty}$ Índice de masa corporal $\left(\mathrm{kg} / \mathrm{m}^{2}\right) ; \pi$ Consumo máximo de oxígeno (ml.kg.min); $\neq$ Sentadilla con salto ( $\mathrm{cm}$ ); $\mu$ Salto contra-movimiento $(\mathrm{cm}) ; ¥$ Fuerza de resistencia abdominal (repeticiones); § Nivel de significancia; a Grupos en los que se identificaron diferencias estadísticamente significativas con la prueba de Tukey.

En la condición física, el grupo de menor edad obtuvo mejores resultados en la flexibilidad y la fuerza de resistencia abdominal, sin diferencias significativas $(p>0,05)$. En cambio, el grupo de mayor edad, evidenció mayores valores en la sentadilla con salto, salto contra-movimiento y el consumo máximo de oxígeno. Solo en esta última variable hubo diferencias estadísticamente significativas $(p<0,05)$, principalmente, al comparar los resultados con el grupo de 14 a 16 años.

Tabla 4. Correlaciones entre las variables analizadas.
En la tabla 4, se observan las correlaciones entre las variables de la composición corporal y el somatotipo, con las variables de la condición física. En ese sentido, se encontró que el IMC tuvo correlación negativa débil con la capacidad aeróbica $(r=-0,389 ; p=0,000)$ y correlación negativa muy débil, con la sentadilla con salto $(r=-0,163 ; p=0,040)$ y el CMJ $(r=-0,157 ; p=0,047)$, es decir, a mayor IMC, menor rendimiento en las variables de condición física y viceversa. Además, se evidenció una correlación positiva muy débil entre el porcentaje de masa residual y la fuerza de resistencia abdominal $(r=0,156 ; p=0,048)$.

\begin{tabular}{|c|c|c|c|c|c|c|}
\hline \multicolumn{2}{|c|}{ Variable } & $\begin{array}{c}\mathrm{VO}_{2 \max } \\
\text { (ml.kg.min) }\end{array}$ & $\begin{array}{c}\text { SJ } \\
(\mathbf{c m})^{\neq}\end{array}$ & $\begin{array}{l}\text { CMJ } \\
(\mathbf{c m})^{\mu}\end{array}$ & $\begin{array}{l}\text { Flexibilidad } \\
\text { (cm) }\end{array}$ & $\begin{array}{c}\text { FRA } \\
\text { (rep) }\end{array}$ \\
\hline \multirow{2}{*}{$\operatorname{IMC}\left(\mathrm{kg} / \mathrm{m}^{2}\right)^{\infty}$} & $r$ & $-0,389^{* *}$ & $-0,163^{*}$ & $-0,157^{*}$ & 0,054 & 0,040 \\
\hline & $p$ & 0,000 & 0,040 & 0,047 & 0,500 & 0,615 \\
\hline \multirow{2}{*}{ Grasa (\%) } & $r$ & $-0,150$ & $-0,135$ & $-0,126$ & $-0,119$ & $-0,139$ \\
\hline & $p$ & 0,058 & 0,088 & 0,112 & 0,134 & 0,079 \\
\hline \multirow{2}{*}{ Músculo (\%) } & $r$ & $-0,020$ & 0,109 & 0,098 & 0,077 & $-0,35$ \\
\hline & $p$ & 0,801 & 0,170 & 0,219 & 0,333 & 0,657 \\
\hline \multirow{2}{*}{ Residual (\%) } & $r$ & $-0,016$ & $-0,020$ & $-0,021$ & 0,050 & $0,156^{*}$ \\
\hline & $p$ & 0,838 & 0,801 & 0,793 & 0,531 & 0,048 \\
\hline \multirow{2}{*}{ Óseo (\%) } & $r$ & 0,059 & 0,010 & 0,020 & 0,033 & $-0,088$ \\
\hline & $p$ & 0,459 & 0,904 & 0,805 & 0,683 & 0,266 \\
\hline \multirow{2}{*}{ Endomorfía } & $r$ & $-0,102$ & $-0,153$ & $-0,148$ & $-0,109$ & $-0,131$ \\
\hline & $p$ & 0,201 & 0,053 & 0,061 & 0,172 & 0,099 \\
\hline \multirow{2}{*}{ Mesomorfía } & $r$ & $-0,230^{* *}$ & 0,134 & 0,141 & $0,211^{* *}$ & $-0,067$ \\
\hline & $p$ & 0,003 & 0,091 & 0,075 & 0,007 & 0,399 \\
\hline \multirow{2}{*}{ Ectomorfía } & $r$ & $0,187^{*}$ & $-0,080$ & $-0,093$ & $-0,263^{* *}$ & $-0,007$ \\
\hline & $p$ & 0,018 & 0,312 & 0,243 & 0,001 & 0,931 \\
\hline
\end{tabular}

$\infty$ Índice de masa corporal $\left(\mathrm{kg} / \mathrm{m}^{2}\right) ; \pi$ Consumo máximo de oxígeno $(\mathrm{ml} . \mathrm{kg} . \mathrm{min}) ;{ }^{\ddagger} \mathrm{SJ}(\mathrm{cm}) ; \mu \mathrm{CMJ}(\mathrm{cm}) ;{ }^{¥}$ Fuerza de resistencia abdominal (repeticiones); p Correlación de Pearson; $r$ Nivel de significancia.

* La correlación es significativa en el nivel 0,05 (bilateral)

** La correlación es significativa en el nivel 0,01 (bilateral) 
También, el componente mesomórfico correlacionó negativamente con la capacidad aeróbica $(r=-0,230 ; p=0,003)$ y positivamente con la flexibilidad $(r=0,211 ; p=0,007)$, mientras que el componente ectomórfico, correlacionó positivamente con el VO2máx $(r=0,187 ; p=0,018)$ y negativamente, con la flexibilidad ( $r=-0,263$; $p=0,001$.

Los resultados obtenidos en el presente estudio indican que las mujeres examinadas tienen un gran desarrollo de la masa muscular y un porcentaje bajo de la masa grasa, evidenciándose un somatotipo predominante de mesomorfia. Las femeninas de mayor edad poseen mejores resultados en la mayoría de pruebas de la condición física; asimismo, poseen un menor IMC y porcentaje graso. Se encontraron algunas correlaciones entre las variables estudiadas, pero estas fueron muy débiles o débiles.

La información y las características de composición corporal, somatotipo y de la condición física recopilados constituyen una información valiosa para los entrenadores deportivos de las diferentes modalidades y ligas, generando una adecuada planificación, programas de nutrición y seguimiento a los entrenamientos.

\section{REFERENCIAS}

1. BALSALOBRE, C.; CAMPO, J.; TEJERO, C.; ALONSO

D. 2012. Relación entre potencia máxima, fuerza máxima, salto vertical y sprint de 30 metros en atletas cuatrocentistas de alto rendimiento. Apunts. Educación Física y Deportes (España). 108:63-69.

https://doi.org/10.5672/apunts.2014-0983.es. (2012/2).108.07

2. CABALLERO, A.; CARRASCO, C.; DE LEÓN, L.; CANDIA, R.; ORTIZ, B. 2019. Somatotipo de mujeres futbolistas universitarias por posición en el terreno de juego. Retos (España). 36(36):228-230.

https://doi.org/10.47197/retos.v36i36.63840

3. CASTIBLANCO, H.; VIDARTE, J.; SÁNCHEZ, J. 2020. Composición corporal y capacidad cardiorrespiratoria en deportistas universitarios de Manizales (Colombia). Nutrición Clínica y Dietética Hospitalaria (España). 40(1):12-19.

https://doi.org/10.12873/401castiblanco
4. GARCÍA, A.; GÓMEZ, M.; ROJAS, J. 2020. Relación entre el índice de masa corporal, índice de masa grasa y tensión arterial en cadetes colombianos con sobrepeso. Archivos de Medicina (España). 20(2):428-436.

https://doi.org/10.30554/arch med.20.2.3535

5. GARCÍA, J.; LÓPEZ, J.; OGANDO, H.; FERNÁNDEZ, A.; PADRÓN, A.; PRIETO, J. 2014. Utilidad de la cineantropometría y la bioimpedancia para orientar la composición corporal y los hábitos de los futbolistas. Retos. (España). 25:117-119. https://doi.org/10.47197/retos.v0i25.34493

6. GONZÁlEZ, M.; MAURO, I.; GARCÍA, B.; FAJARDO, D.; GARICANO, E. 2015. Valoración nutricional, evaluación de la composición corporal y su relación con el rendimiento deportivo en un equipo de fútbol femenino María González-Neira. Revista Española de Nutrición Humana y Dietetica. 19(1):36-48. https://doi.org/10.14306/renhyd.19.1.109

7. GONZÁLEZ, J.; VARGAS, A.; FERNÁNDEZ, J.; GONZÁLEZ, A.; GÓMEZ, R.; COSTA, J. 2011. Original análisis del baile flamenco: cargas de trabajo y condición fisica physical assesment in flamenco dance. Revista Inernacional de Medicina y Ciencias de la Actividad Física y del Deporte (España). 11(44):708-720.

8. GUTIÉRREZ, L.; ZAVALA, J.; FUENTES, C.; YÁÑEZ, R. 2020. Características Antropométricas y Somatotipo en Seleccionados Chilenos de Remo Anthropometric Characteristics and Somatotype in Elite Chilean Rowers. International Journal of Morphology (Chile). 38(1):114-119. http://dx.doi.org/10.4067/S0717-9502 2020000100114

9. INTERNATIONAL SOCIETY FOR THE ADVANCEMENT OF KINANTHROPOMETRY, ISAK. 2001. Normas Internacionales para la Valoración Antropométrica. Librería Nacional de Australia. 77p.

10. IRIGOYEN, J.Y.; ARCOS LARUMBE, A. 2013. Evolución del rendimiento aeróbico y anaeróbico en futbolistas profesionales tras la pretemporada. Cultura, Ciencia y Deporte. 8(24):207-215. 
11. MARTÍNEZ, C.; VEIGA, C.; LÓPEZ, A.; COBO, J.; CARBAJAL, A. 2005. Evaluación del estado nutricional de un grupo de estudiantes universitarios mediante parámetros dietéticos y de composición corporal. Nutrición Hospitalaria (España). 20(3):197-203.

12. MINISTERIO DE SALUD. 1993. Resolución número 8430 de 1993 (octubre 4). Por la cual se establecen las normas científicas, técnicas y administrativas para la investigación en salud. Disponible desde Internet en: https://www.minsalud.gov. co/sites/rid/Lists/BibliotecaDigital/RIDE/ DE/DIJ/RESOLUCION-8430-DE-1993.PDF

13. MORO, P.; BUCES, M.; GONZÁLEZ, M.; DE ESPINOSA, M.; VELASCO, E.; LÓPEZ, N.; DOLORES, M.; SERRANO, M. 2016. Semilongitudinal analysis of physical status in madrilenian adolescents. Archivos de Medicina del Deporte(España).33(3):183-192.

14. NUTRITION AND HEALTH TRANSITION IN THE DEVELOPING WORLD: THE

TIME TO ACT. 2002. Public Health Nutrition (Inglaterra). 5(1):279-280.

15. PALOMINO, C.; OTERO, F.; GONZÁLEZ, J. 2016. Análisis de la adiposidad y la condición física en escolares colombianos. Biomédica (Colombia) 36(3):343-353. https://doi. org/10.7705/biomedica.v36i3.3148

16. PALOMINO, C.; REYES, F.; SÁNCHEZ, A. 2018. Niveles de actividad física, calidad de vida relaciona con la salud, autoconcepto físico e índice de masa corporal: un estudio en escolares colombianos. Biomédica (Colombia) 38(2):224-231. https://doi. org /10.7705/biomedica.v38i0.3964

17. RAMOS, C.; GÓMEZ, M. 2018. Valoración de la Condición Física y Prescripción del Ejercicio Físico. Editorial Universidad del Tolima (Colombia). 312p.

18. RIVERA, I.; FLOODY, M.; DELGADO, P.; 25. SCHIFFERLI, I.; OSORIO, A.; MARTÍNEZ, C. 2017. Nutritional status, cardiovascular health, VO2 max and habits in university students: A comparison between two health promotion careers. Revista Facultad de Medicina (Colombia). 65(3):447-451. https:// doi.org/10.15446/revfacmed.v65n3.55185

19. SÁNCHEZ, B.; SALAS, J. 2009. Determinación del consumo máximo de oxígeno del futbolista costarricense de primera división en pretemporada 2008. MHSalud: Revista En Ciencias Del Movimiento Humano y Salud (Costa Rica). 6(2):1-5. https://doi. org/10.15359/mhs.6-2.2

20. SEDANO, S.; CUADRADO, G.; REDONDO, J.; DE BENITO, A. 2009. Perfil antropométrico de las mujeres futbolistas españolas. Análisis en función del nivel competitivo y de la posición ocupada habitualmente en el terreno de juego. Apunts. (España). 4(98):78-87.

21. SILVA, C.; TORRES, L.; RAHAL, A.; TERRA, J.; VIANNA, E. 2006. Comparison of morning and afternoon exercise training for asthmatic children. Brazilian Journal of Medical and Biological Research. 39(1):71-78. http://dx.doi. org/10.1590/s0100-879X2006000100008

22. STERKOWICZ, K.; STERKOWICZ, S.; BISKUP, L.; ŻARÓW, R.; KRYST, Ł.; OZIMEK, M. 2019. Somatotype, body composition, and physical fitness in artistic gymnasts depending on age and preferred event. PLOS ONE (Estados Unidos). 14(2). https:// doi.org/10.1371/journal.pone.0211533

23. TANAKA, H.; MONAHAN, K.; SEALS, D. 2001. Age-predicted maximal heart rate revisited. Journal of the American College of Cardiology (España). 37(1):153-156. https://doi org /10.1016/S0735-1097(00)01054-8

24. WELLS, K.; DILLON, E. 1952. The sit and reach $-a$ test of back and leg flexibility. Research Quarterly of the American Association for Health, Physical Education and Recreation. 23(1):115-118. https:// doi.org/10.1080/10671188.1952.10761965

WORLD MEDICAL ASSOCIATION, WMA. 2017. Declaración de Helsinki de la AMM Principios éticos para las investigaciones médicas en seres humanos. 